\title{
Caracterización de la microbiota levaduriforme residente en la vagina de perras en diferentes fases del ciclo estral
}

\author{
Characterization of vaginal microbiotic yeasts of bitches in distinct stages of the oestrous cycle \\ M B Cleff ${ }^{* 1}$, M O Xavier', A A Martins², R Santin², M C A Meireles ${ }^{3}$ \\ ${ }^{\mathbf{1}}$ Aluno de Mestrado do Programa de Pós-Graduação em Veterinária, Faculdade de Veterinária (FAVET), \\ Universidade Federal de Pelotas (UFPel), Pelotas, RS Brasil. \\ ${ }^{2}$ Aluno de Iniciação Científica, Departamento de Veterinária Preventiva, FAVET - UFPel. \\ ${ }^{3}$ Doutor, Prof. Departamento Veterinária Preventiva, FAVET - UFPel.
}

\begin{abstract}
SUMMARY
Yeasts are commensal organisms frequently isolated from the skin and mucous membranes of domestic and wild mammals. Although yeasts are frequently isolated from women's vaginal mucus, similar studies in canine are rare, therefore their behaviour during the canine oestrous cycle is unknown. The purpose of this study was to isolate yeasts from the vaginal microbiota of bitches and to evaluate their behaviour in different stages of the oestrous cycle. We examined 75 samples of mucus from the vaginal cavity of 75 domestic bitches and 224 samples from 14 experimental bitches. The stages of the oestrous cycle were characterized through vaginal cytology and clinical observation of bleeding, vulvar oedema and pucker. Samples were examined visually, and also cultured in Sabouraud dextrose agar with chloranphenicol, at $37^{\circ} \mathrm{C}$ for 10 days. The yeasts were classified according to their macro and micro morphology, biochemical tests, germination tube and micro culture. Yeasts were isolated from 25 (33.3\%) samples from domestic females. Among the samples collected during proestrous, oestrous, diestrous and anestrous, $56.3 \% 16.7 \% 66.7 \%$ and $20.5 \%$ were positive, respectively. Yeasts were isolated from $147(65.6 \%)$ samples from experimental bitches. Among the samples collected during proestrous, oestrous, diestrous and anestrous, positive samples represented $61.9 \%, 71.9 \%, 82.7 \%$, and 57.1\%, respectively. The identified species were Candida spp, M. pachydermatis and Rhodotorula spp, which are commonly present in the vaginal mucus microbiota of healthy bitches and their frequency changes with the different stages of the oestrous cycle.
\end{abstract}

Palabras clave: levaduras, vagina, perras.

Key words: yeasts, vagina, bitches.

\section{INTRODUCCION}

En las últimas décadas las enfermedades fúngicas pasaron a tener mayor importancia para la salud pública, principalmente debido al mayor número de individuos con microbiota alterada o inmunosuprimidos, que son más propensos a desenvolver infecciones por hongos oportunistas, como en el caso de levadurosis (Plant y col 1992, Guillot y col 1996, Raposo y col 1996, Sierra y col 2000, Silva y col 2002). Las levaduras son integrantes de la microbiota vaginal de diferentes especies, pero todavía los factores que favorecen su proliferación y transmisión de un microorganismo comensal para patógeno son poco comprendidos (Bond y col 1995, Sidrim y Moreira 1999,

Aceptado: 29.08.2006

* Correspondencia: Universidade Federal de Pelotas, Faculdade de Veterinária, Departamento de Veterinária Preventiva, Laboratório de Micologia-Campus, Capão do Leão, Pelotas, Brasil.CEP: 96010.900. E-mail: mbcleff@brturbo.com.br / meireles@ufpel. tche.br

** Fuente de Financiamiento: CAPES e CNPq.
Sierra y col 2000, Ribeiro y col 2001). En su mayoría las vaginitis en perras son tratadas sin el conocimiento exacto de los patógenos involucrados, no siendo por ello registrados los casos de las afecciones vaginales producidas por levaduras. La presencia de los hongos puede tornarse un problema clínico, especialmente en los inmunocomprometidos (Ribeiro y col 2001, Lacaz y col 2002), debiendo el veterinario tener especial atención con los pacientes con historia de vaginitis reincidentes y/o que presentan problemas reproductivos.

En la especie canina, el ciclo estral está caracterizado por innumerables alteraciones, mediadas por hormonas, que inducen a modificaciones morfológicas, clínicas, citológicas y del tracto reproductivo, específicamente en la vagina (Mialot 1988, Bjurstörm y Linde-Forsberg 1992, Feldman y Nelson 1996, Watts y col 1996, Oliveira y col 1998). Factores estresantes como limitación en el ejercicio, peleas por territorio y períodos de celo, entre otros, asociados con las alteraciones hormonales ocurridas durante el ciclo pueden determinar alteraciones de los mecanismos físicos, químicos o inmunológicos normales, que limitan la colonización microbiana de la piel y las mucosas (Plant y col 1992, Bond 1997, Sierra y col 
2000, Wira y col 2000, Ziarrusta 2002). Aunque existen importantes estudios sobre las fases del ciclo estral canino (Concannon y col 1989, Feldman y Nelson 1996 , Vannucchi y col 1997, Rodrigues y Rodrigues 2002) todavía no se ha determinado la relación entre las alteraciones fisiológicas ocurridas durante el ciclo y la colonización de levaduras en la mucosa vaginal, las cuales en pacientes humanos tienen gran importancia (Fidel Jr. y col 2000, Ziarrusta 2002, Fidel Jr. y col 2004). En este estudio se buscó determinar las fases del ciclo estral, aislar e identificar levaduras de la mucosa vaginal y establecer si hay alteración de la microbiota levaduriforme y la frecuencia de aislamiento en las diferentes fases del ciclo estral de perras.

\section{MATERIAL Y METODOS}

En un período de 10 meses, comprendidos entre marzo y diciembre de 2003, en la ciudad de Pelotas-RS-Brasil, fue estudiada la presencia de levaduras en la mucosa vaginal de 89 perras en las diferentes fases del ciclo reproductivo, las cuales fueron distribuidas en dos grupos distintos, de acuerdo con el origen del animal, siendo identificados como Grupo 1 (perras caseras) y Grupo 2 (perras del canil experimental del Hospital de Clínicas Veterinaria (HCV) - Facultad de Veterinária (FAVET) Universidad Federal de Pelotas - UFPel).

Grupo 1: Inicialmente los animales de este grupo fueron identificados por edad y raza, siendo seleccionados aquellos mestizos con edad entre 2-5 años, vacunados y desparasitados regularmente, y que no hubieran sido medicados con drogas inmunosupresoras en los últimos seis meses. Fueron estudiadas 75 perras, las cuales fueron sometidas a examen clínico general y del sistema reproductivo, a fin de auxiliar en la identificación de la fase del ciclo estral, realizando una colecta de cada hembra, independiente de la fase del ciclo en que se encontraban, resultando en 75 muestras de la cavidad vaginal.

Grupo 2: Los animales pertenecientes a este grupo eran 14 hembras, mestizas, provenientes del Bioterio Central de la UFPel, siendo todos vacunados ${ }^{1}$ y despara$\operatorname{sitados}^{2}$ en el inicio del experimento. Por un período de diez meses, los animales fueron alojados en el canil de investigación (HCV- UFPel), que está compuesto por boxes, subdividiendo en área interna y externa o solario. Durante ese período las hembras tuvieron el ejercicio restringido, no se hizo uso de medicamentos, recibieron ración concentrada, en cantidad de acuerdo con el peso corporal y agua "ad-libitum". Las muestras fueron recogidas luego de un período inicial de adaptación

(®) Pfizer Saúde Animal - Vanguard ${ }^{\circledR}$ HTLP 5/CV-L.

2 (B - Agribrands do Brasil Ltda - Canex composto (Palmoato de Pirantel, Praziquantel) de los animales, resultando 16 colectas por hembra, de un total de 224 muestras del material recogido de la vagina.

Todos los animales (Grupo 1 y 2) fueron acompañados con fichas de identificación, que contenían información referida a la condición anatómica vulvar de las perras, presencia o ausencia de secreciones, comportamiento del animal, higiene ambiental y de los animales, acceso a la calle y si convivían o no con otros animales.

Recolección de las muestras. En los dos grupos las muestras vaginales fueron recogidas con hisopos y cureta. Las muestras obtenidas con hisopo fueron utilizadas para la realización de examen citovaginal y micológico directo, y las recolectadas con cureta para aislamiento de levaduras. Anterior a la colecta, se realizó limpieza de la región vulvar con solución fisiológica, seguida de secado con papel toalla. Con el auxilio de un espéculo vaginal, el hisopo humedecido en solución salina estéril $(0,9 \%)$ fue introducido por la comisura dorsal de la vulva en dirección a la región dorso-craneal de la vagina y friccionada en las paredes dorso-lateral, siendo inmediatamente realizado el mismo procedimiento con la cureta, obteniéndose así raspados de las paredes vaginales.

Examen citovaginal. Para la determinación de las fases del ciclo estral de las perras se utilizó extendidos vaginales seriados, coloreados por el método de Giemsa. La interpretación del extendido se basó en la morfología de las células epiteliales, dándose especial atención a las células nucleadas (superficiales, intermediarias y parabasal), células sin núcleo o con vestigio nuclear (cornificadas), leucocitos, eritrocitos y cantidad de mucus.

Cultivo. Inmediatamente luego de la obtención, las muestras fueron sembradas por extensión en placa con agarSabouraud dextrosa con cloranfenicol e incubadas a $37^{\circ} \mathrm{C}$ por 10 días, para análisis macro y micromorfológico de las colonias.

Clasificación de las levaduras. Las colonias levaduriformes fueron caracterizadas por macro y micromorfología, pruebas bioquímicas, prueba de tubo germinativo y microcultivo en agar fubá. La clasificación de las levaduras del género Candida y Rhodoturola fue realizada según Lacaz 2002 y el de Malassezia por Guillot 1996.

Análisis estadísticos. Los datos fueron analizados utilizándose el programa Statistix 7.0 (Statistix 2000), a través de la distribución de las frecuencias de levaduras en las diferentes fases del ciclo reproductivo. Las pruebas paramétricas de X cuadrado fueron utilizadas para comparación de los aislamientos de levaduras en las diferentes fases del ciclo estral, y el test de Fisher fue utilizado en el análisis de los resultados de la colecta en animales caseros. 


\section{RESULTADOS Y DISCUSION}

De las 75 muestras obtenidas de la cavidad vaginal de las hembras del Grupo 1, 16 estaban en el período de proestro, 6 en estro, 9 en diestro y 44 en anestro. El aislamiento fúngico ocurrió en 25 muestras (33,3\%), siendo las levaduras clasificadas como Candida spp, $M$. pachydermatis y Rhodotorula spp. De las 224 muestras recogidas de las hembras del Grupo 2, 21 fueron colectadas en la fase de proestro, 32 en estro, 52 en diestro y 119 en anestro. El aislamiento de levaduras ocurrió en 147 muestras $(65,6 \%)$, siendo las levaduras clasificadas como Candida spp, M. pachydermatis y Rhodotorula spp, las que se prensentaron en monocultivo y/o asociadas. Para el cálculo de las frecuencias de levaduras fueron considerados los valores relativos al total de las muestras obtenidas en cada fase del ciclo.

El experimento fue realizado en animales caseros (Grupo 1) en los cuales la obtención de muestras fue aleatoria y realizada una sola vez, y en animales experimentales (Grupo 2) que fueron confinados y la toma de muestras realizada periódicamente en el mismo animal, con el acompañamiento de las fases del ciclo estral. La mayor tasa $(65,6 \%)$ de aislamientos de levaduras del Grupo 2 en relación al Grupo $1(33,3 \%)$ puede haber ocurrido, debido al largo período en que los animales estuvieron confinados con limitación del ejercicio, episodios de celos ocasionando períodos de estrés, lo que podría facilitar la proliferación de levaduras (Bond 1997, Rodríguez y col 2003). Además se debe considerar el mayor número de muestras realizadas en estos animales, lo que permitió un mejor análisis de la microbiota levaduriforme en este grupo.
En las hembras del Grupo 1, la fase en que se obtuvo mayor frecuencia de aislamiento fue el diestro $(66,7 \%)$, seguido de proestro $(56,3 \%)$, siendo la diferencia entre las fases estadísticamente significativa $(\mathrm{P}<0,05)$. Considerando los aislamientos obtenidos durante todas las fases del ciclo estral, $M$. pachydermatis ocurrió en monocultivo en 10 muestras $(13,3 \%)$ y asociada a Candida spp en ocho (10,7\%), en tanto en la fase de estro no hubo aislamiento de esta levadura. El aislamiento de Candida spp fue mayor en asociación con $M$. pachydermatis ( $\mathrm{n}=8 / 10,7 \%)$ y Rhodotorula $\mathrm{spp}(\mathrm{n}=2 / 2,7 \%)$, presentándose en monocultivo en dos muestras $(2,6 \%)$. Rhodotorula spp fue aislada de cinco muestras, en dos oportunidades $(2,7 \%)$ estuvo asociada a Candida spp (cuadro 1).

En las hembras del Grupo 2, la fase del ciclo estral, en la cual ocurrió mayor frecuencia de aislamiento fue en el diestro $(82,7 \%)$ y estro $(71,9 \%)$ con una diferencia estadísticamente significativa en cuanto a aislamiento de levaduras $(\mathrm{P}<0,05)$. Considerando el aislamiento concomitante de levaduras, el género Candida obtuvo mayor aislamiento en diestro, $M$. pachydermatis en anestro y diestro, mientras que Rhodotorula spp en la fase de estro. En cuanto a la frecuencia de aislamiento en monocultivo es $21,5 \%$ cuando está asociada a $M$. pachydermatis y/o Rhodotorula spp. M. pachydermatis se presentó en monocultivo en $17,7 \%$ de las muestras y asociada con otras levaduras en $28,6 \%$. La mayor frecuencia de Rhodotorula spp (14,3\%) fue observada estando asociada a Candida spp y/o M. pachydermatis, en tanto el aislamiento en monocultivo ocurrió en un $1,8 \%$ de las muestras (cuadro 2).

Cuadro 1. Frecuencia de levaduras en relación a las fases del ciclo estral en 75 muestras obtenidas de la vagina de perras (caniles particulares/Pelotas-RS-Brasil).

Frequency of yeasts on 75 samples of mucus from the vaginal cavity of domestic bitches in different stages of the oestrous cycle (FAVET/UFPel-RS-Brasil).

\begin{tabular}{|c|c|c|c|c|c|c|c|c|c|c|}
\hline \multirow{3}{*}{ Levaduras } & \multicolumn{10}{|c|}{ Fases del ciclo estral } \\
\hline & \multicolumn{2}{|c|}{ Proestro } & \multicolumn{2}{|c|}{ Estro } & \multicolumn{2}{|c|}{ Diestro } & \multicolumn{2}{|c|}{ Anestro } & \multicolumn{2}{|c|}{ Total } \\
\hline & $\mathrm{N}^{\mathrm{o}}$ & $(\%)$ & $\mathrm{N}^{\circ}$ & $(\%)$ & $\mathrm{N}^{\circ}$ & $(\%)$ & $\mathrm{N}^{\mathrm{o}}$ & $(\%)$ & $\mathrm{N}^{\mathrm{o}}$ & $(\%)$ \\
\hline Candida spp & - & - & 1 & $(16,7)$ & - & - & 1 & $(2,3)$ & 2 & $(2,6)$ \\
\hline Malassezia pachydermatis & 4 & $(25)$ & - & - & 2 & $(22,2)$ & 4 & $(9,1)$ & 10 & $(13,3)$ \\
\hline Rhodotorula spp & 2 & $(12,5)$ & - & - & - & - & 1 & $(2,3)$ & 3 & $(4,0)$ \\
\hline Candida $\mathrm{spp} / M$. pachydermatis & 3 & $(18,8)$ & - & - & 2 & $(22,2)$ & 3 & $(6,8)$ & 8 & $(10,7)$ \\
\hline Candida spp/Rhodotorula spp & - & - & - & - & 2 & $(22,2)$ & - & - & 2 & $(2,7)$ \\
\hline Positivo & 9 & $(56,3)^{\mathrm{b}}$ & 1 & $(16,7)^{\mathrm{d}}$ & 6 & $(66,6)^{\mathrm{a}}$ & 9 & $(20,5) \mathrm{c}$ & 25 & $(33,3)$ \\
\hline Negativo & 7 & $(43,7)$ & 5 & $(83,3)$ & 3 & $(33,4)$ & 35 & $(79,5)$ & 50 & $(66,7)$ \\
\hline Total & 16 & $(100)$ & 6 & $(100)$ & 9 & $(100)$ & 44 & $(100)$ & 75 & $(100)$ \\
\hline
\end{tabular}

$\mathrm{n}=$ número absoluto; $(\%)=$ porcentual; $* \mathrm{P}<0,05 ;{ }^{\mathrm{a}, \mathrm{b}, \mathrm{c}, \mathrm{d}}$ Exponentes diferentes en la misma línea difieren por lo menos $\mathrm{P}<0,05$. 
Cuadro 2. Frecuencia de levaduras en relación a las fases del ciclo estral de las 224 muestras de la vagina de 14 perras (caniles experimentales-FAVET/UFPel).

Frequency of yeasts on 224 samples of mucus from the vaginal cavity of 14 experimental bitches in different stages of the oestrous cycle (FAVET/UFPel).

Fases del ciclo estral

\begin{tabular}{|c|c|c|c|c|c|c|c|c|c|c|}
\hline \multirow[t]{2}{*}{ Levaduras } & \multicolumn{2}{|c|}{ Proestro } & \multicolumn{2}{|c|}{ Estro } & \multicolumn{2}{|c|}{ Diestro } & \multicolumn{2}{|c|}{ Anestro } & \multicolumn{2}{|c|}{ Total } \\
\hline & $\mathrm{N}^{\mathrm{o}}$ & $(\%)$ & $\mathrm{N}^{\mathrm{o}}$ & $(\%)$ & $\mathrm{N}^{\mathrm{o}}$ & $(\%)$ & $\mathrm{N}^{\mathrm{o}}$ & $(\%)$ & $\mathrm{N}^{\mathrm{o}}$ & $(\%)$ \\
\hline Candida spp & 4 & (19) & 2 & $(6,3)$ & 14 & $(26,9)$ & 15 & $(12,6)$ & 35 & $(15,6)$ \\
\hline Malassezia pachydermatis & 3 & $(14,3)$ & 3 & $(9,4)$ & 9 & $(17,3)$ & 25 & $(21)$ & 40 & $(17,8)$ \\
\hline Rhodotorula spp & - & - & 2 & $(6,3)$ & 1 & $(1,9)$ & 1 & $(0,84)$ & 4 & $(1,8)$ \\
\hline Candida spp/M. pachydermatis & 2 & $(9,5)$ & 9 & $(28,1)$ & 13 & $(25)$ & 12 & (10) & 36 & $(16,1)$ \\
\hline Candida spp/Rhodotorula spp & 1 & $(4,8)$ & - & - & 1 & $(1,9)$ & 2 & $(1,8)$ & 4 & $(1,8)$ \\
\hline M. pachydermatis/ & & & & & & & & & & \\
\hline Rhodotorula spp & 1 & $(4,8)$ & 4 & $(12,5)$ & 2 & $(3,9)$ & 13 & $(10,9)$ & 20 & $(8,9)$ \\
\hline M.pachydermatis/ & & & & & & & & & & \\
\hline Rhodotorula/Candida spp & 2 & $(9,5)$ & 3 & $(9,4)$ & 3 & $(5,8)$ & - & - & 8 & $(3,6)$ \\
\hline Positivo & 13 & $(61,9)^{\mathrm{bc}}$ & 23 & $(71,9)^{\mathrm{ab}}$ & 43 & $(82,7)^{\mathrm{a}}$ & 68 & $(57,1)^{\mathrm{c}}$ & 147 & $(65,6)$ \\
\hline Negativo & 8 & $(38,1)$ & 9 & $(28,1)$ & 9 & $(17,3)$ & 51 & $(42,9)$ & 77 & $(34,4)$ \\
\hline Total & 21 & (100) & 32 & (100) & 52 & (100) & 119 & (100) & 224 & (100) \\
\hline
\end{tabular}

$\mathrm{n}=$ número absoluto; $(\%)=$ porcentual; $* \mathrm{P}<0,05 ;{ }^{\mathrm{a}, \mathrm{b}, \mathrm{c}}$ Exponentes diferentes en la misma línea difieren por lo menos $\mathrm{P}<0,05$.

Las levaduras del género Candida fueron aisladas tanto de las muestras obtenidas del Grupo 1 como del Grupo 2, principalmente en las fases de diestro y proestro, en animales clínicamente sanos, sin síntomas de candidiasis vaginal. Sin embargo hay diferencias en relación al ciclo reproductivo de hembras caninas, que son monoestras estacionales y la mujer que cicla mensualmente. Los resultados obtenidos en relación al aislamiento de Candida spp de la cavidad vaginal de hembras caninas sanas ( $16 \%$ y $36 \%$, respectivamente) fueron semejantes a los observados en el mismo sitio anatómico en mujeres sanas, cuya frecuencia de aislamiento de este género fue de 20 a 30\% (Linares y Marín 1978, Ziarrusta 2002), pudiendo llegar al 48\% (Abu-Elteen y col 1997). En mujeres, el género Candida ha sido comúnmente aislado de la mucosa vaginal sana (Linares y Marín 1978, Abu-Elteen y col 1997, Ribeiro y col 2001, Ziarrusta 2002) y en mayor frecuencia en los casos de vaginitis (Fidel Jr y col 2000, Fahey y Wira 2002, Ziarrusta 2002), por eso en caninos no fueron encontrados relatos de vaginitis por Candida, así como estudios en relación a su presencia en la mucosa vaginal de animales sanos, aunque ya había sido aislada de otros sitios anatómicos, como conducto auditivo externo (Feijó y col 1997, Bornand 1992) piel (Guillot y col 1994, Raposo y col 1996, Moretti y col 2004, Moretti y col 2006), mucosa nasal, oral y anal (Greene y Chandler 1998).

La frecuencia de $M$. pachydermatis observada en la mucosa vaginal de las perras (24 y 47\%) fue notoriamente mayor que la demostrada por Bond (1995) y
Oliveira y col (1998), quienes al estudiar la microbiota vaginal obtuvieron frecuencias en torno de $8 \%$ sin relacionarla con el ciclo estral. El microclima caliente y húmedo de la vagina puede haber facilitado la multiplicación de las células de $M$. pachydermatis, como ya fue observado en otros sitios anatómicos como meato acústico externo en caninos (Duffait 1983, Baptista 1984, Bond y col 1995, Carlotti 1997, Bond y col 2000). M. pachydermatis fue observada con frecuencia variando entre $53 \%$ a $91 \%$ en muestras del meato acústico sano de canes, y en $35 \%$ en las muestras del tegumento cutáneo sano de perros (Bond y col 1995, Carlotti 1997, Bond y col 2000, Nascente 2001).

Durante el estudio, el aislamiento de Rhodotorula spp fue mayor cuando estuvo asociado con Candida spp y con M. pachydermatis. La asociación de Rhodotorula spp con otras levaduras, como Candida spp, Malassezia pachydermatis, Cryptococcus neoformans ha sido descrita por otros autores (Guillot y col 1994). Rhodotorula spp fue también aislada simultáneamente con otras levaduras en la piel de canes (Guillot y col 1994) y en el meato acústico externo (Bornand 1992). Rhodotorula spp es descrita como habitante del suelo y aire, lo que facilitaría la colonización de las áreas de piel y mucosas de los animales (Bornand 1992, Biswas y col 2001). En los animales alojados en el canil experimental (Grupo 2) la mayor frecuencia ocurrió en la fase de estro (cuadro 2), posiblemente en función del relajamiento de la vagina, lo que podría facilitar la penetración de microorganismos del ambiente. Esta levadura no ha sido mencionada en el 
tracto reproductivo de animales y humanos, aunque haya sido detectada en infecciones urinarias, principalmente en pacientes humanos inmunodeprimidos (Rusthoven y col 1984, Rippon 1988, Guerra y col 1992, Hazen 1995, Gyaurgieva y col 1996, Lui y col 1998, Papadogeorgakis y col 1999).

En relación al ciclo reproductivo de las hembras caninas, en este estudio el aislamiento de levaduras de la mucosa vaginal sufre alteraciones de acuerdo con los cambios en las fases del ciclo estral, los cuales, según observaciones de Roszel (1975), Jöchle y Andersen (1977), Linde y Karlasson (1984), Mialot (1988), Concannon y col (1989), Valtonen y Jalkanen (1993), Feldman y Nelson (1996) conducen a cambios morfológicos, clínicos y citológicos en la mucosa vaginal.

En la fase de diestro fue observada una mayor frecuencia de aislamiento de levaduras en comparación con el proestro y estro, en que siempre hubo aislamiento, aunque en menor frecuencia, mientras en anestro fue la fase de menor aislamiento. Específicamente el aislamiento de levaduras del género Candida y de Malassezia pachydermatis fue mayor durante la fase de diestro y reducida en anestro, aunque Rhodotorula spp tuvo mayor aislamiento en la fase de estro. Los altos niveles de aislamiento en la fase de diestro, probablemente, se relacionan a los niveles elevados de progesterona (Jeffcoate 1998, Verstegen 1999), la cual actúa como supresor de la inmunidad celular (Ziarrusta 2002).

Las frecuencias observadas en la fase de proestro ocurren, probablemente, debido al aumento de los niveles de estrógeno (Jöchle y Andersen 1977, Olson y Nett 1986, Valtonen y Jalkanen 1993), los cuales actúan propiciando la colonización fúngica, debido al incremento de la exposición de los complejos epiteliales glucoproteicos, que actúan como receptores de los agentes fúngicos (Ziarrusta 2002). Según Hafez (1995), la acción estrogénica acarrea el acúmulo de glucógeno, que cuando es metabolizado se transforma en glucosa y consecuentemente en ácido láctico, bajando el pH vaginal, favoreciendo con eso el desarrollo de los lactobacilos y levaduras (Lacaz y col 2002). De acuerdo con Oliveira y col (1998), en perras sanas el pH vaginal en la fase de proestro se encuentra ácido; otro aspecto que podría influenciar la presencia de levaduras en esta fase sería una disminución de inmunoglobulinas $\mathrm{G}$ y A por los altos niveles de estrógeno (Wira y col 2000). En el estro, en que los niveles de estrógeno se encuentran aumentados, también se obtuvo aislamiento de levaduras. En modelos animales las funciones inmunes uterinas aumentan con la presencia de estrógeno y progesterona, mientras que las vaginales se encuentran reducidas (Wira y col 2000). En la fase de anestro la frecuencia de levaduras aisladas de la cavidad vaginal de las hembras caninas fue menor, probablemente debido a que las concentraciones hormonales permanecen a un nivel basal (Concannon y col 1989, Valtonen y Jalkanen 1993, Feldman y Nelson 1996), o debido a que el $\mathrm{pH}$ vaginal se encuentra alcalino. Según Oliveira y col (1998), el pH alcalino favorecería el desarrollo de agentes microbianos, los cuales podrían suprimir la flora fúngica, justificando la baja frecuencia de levaduras aisladas.

Debido a la sobrevida de los animales de compañía, es cada vez más frecuente la presencia de pacientes geriátricos, inmunocomprometidos y/o con multiples disfunciones en la clínica, que podrían tornarse susceptibles a las infecciones oportunistas (Moretti y col 2004, Moretti y col 2006). Además, la relación entre los animales de estimación y sus proprietarios se tornan bastante estrechas, exigiendo de los clínicos veterinarios la búsqueda de diagnósticos más precisos y una conducta terapéutica diferenciada, a fin de ofrecer una mejor calidad de vida de esos pacientes. El diagnóstico micológico deberá ser hecho a través de recolección y análisis del material, con una apropiada identificación de la especie fúngica involucrada, a fin de prescribir la terapia antifúngica adecuada.

Los resultados obtenidos permiten concluir que Candida spp, Rhodotorula spp y M. pachydermatis son parte integrante de la microbiota vaginal de perras sanas, constatándose que la fase del ciclo estral interfiere en la frecuencia de levaduras aisladas de la vagina de perras, siendo más frecuente durante el diestro y menos frecuente en el anestro.

\section{RESUMEN}

Hongos levaduriformes son aislados frecuentemente de los animales domésticos y silvestres, siendo algunos comensales del tegumento y mucosas. Las levaduras son frecuentes en la mucosa vaginal de las mujeres, pero en perras son escasos los estudios referentes a la microbiota fúngica vaginal, sin conocerse su relación con el ciclo reproductivo. En este estudio se buscó aislar e identificar levaduras de la microbiota vaginal de perras, y establecer si hay alteración de la microbiota levaduriforme en las fases del ciclo reproductivo. Se analizaron 75 muestras de la vagina de 75 perras de los caniles particulares (Grupo I) y 224 muestras de 14 perras experimentales (Grupo II). El ciclo estral fue determinado por citología vaginal y se registraron observaciones clínicas como sangrado, edema y pliegues de vulva. Las muestras fueron sometidas a examen directo y cultivos en Agar-Sabouraud dextrosa con cloranfenicol, a $37^{\circ} \mathrm{C}$ por 10 días. Las levaduras fueron clasificadas por macro y micromorfología, pruebas bioquímicas, tubo germinativo y microcultivo. De las 75 muestras del grupo I, el aislamiento fue $33,3 \%(\mathrm{n}=25)$; las muestras fueron obtenidas en las fases de proestro $56,3 \%$, estro $16,7 \%$, diestro $66,7 \%$ y anestro $20,5 \%$, respectivamente. De las 224 muestras del grupo II se aislaron levaduras en 147 (65,6\%): en proestro $61,9 \%$, estro $71,9 \%$, diestro $82,7 \%$ y anestro $57,1 \%$, respectivamente. Las levaduras fueron identificadas como Candida spp, Malassezia pachydermatis y Rhodoturola spp. Los resultados sugieren que estas levaduras forman parte de la microbiota vaginal de perras sanas y sus frecuencias se alteran en las fases del ciclo reproductivo.

\section{REFERENCIAS}

Abu-Elteen K H, N Malek, N A A Amma Wahid. 1997. Prevalence and susceptibility of vaginal yeast isolates in Jordan. Mycoses 40,179-185. 
Baptista G. 1984. Incidência, características morfológicas, fisiológicas e antigênicas de levaduras do gênero Malassezia. Tese Doutorado, Pós-Graduação em Microbiologia e Imunologia, Escola Paulista de Medicina.

Biswas S K, K Yokoyama, K Nishimura, M Miyaji. 2001. Molecular phylogenetics of the genus Rhodotorula and related basidiomycetous yeasts inferred from the mitochondrial cytochrome b gene. Inter J System Evolut Microb 51, 1191-1199.

Bjurstörm L, C Linde-Forsberg. 1992. Study of aerobic flora of the genital tract in the breeding bitches. Am J Vet Res 53, 665-669.

Bond R, L E M Saijonmaa-Koulumies, D H Lloyd. 1995. Population sizes and frequency of Malassezia pachydermatis at skin and mucosal sites on healthy dogs. J Small An Pract 36, 147-150.

Bond R 1997. Malassezia pachydermatis y enfermedad dermatológica canina. Waltham Focus 7, 27-31.

Bond R, A I Lamport, D Lloyd. 2000. Colonisation status of Malassezia pachydermatis on the hair and in the hair follicle of healthy beagle dogs. Res Vet Sci 68, 291-293.

Bornand V. 1992. Bactériologie et mycologie de l'otite externe du chien. Schweiz Arch Tierheilk 134, 1-8.

Carlotti D N. 1997. Canine and feline superficial fungal skin infections. Vet Quart 19, 45 - 46.

Concannon P W, J P McCann, M Temple. 1989. Biology and endocrinology of ovulation, pregnancy and parturition in the dog. J Repr Fert supplem 39, 3-25.

Dufait R. 1983. Presence de M. pachydermatis (Syn. Pityrosporum canis) sur les poils et les plumes d'animaux domestiques. Bull Soc Franc Mycol Med 14, 19-22.

Fahey J V, C R Wira. 2002. Effect of menstrual status on antibacterial activity and secretory leukocyte protease inhibitor production by human uterine epithelial cells in culture. J Infect Dis 185, 1606-1613.

Feijó F M C, S G Campos, R H R Ramadinha. 1997. Características de Candida tropicalis como patógeno em otites. Anais XXV Congresso Brasileiro de Medicina Veterinária, Gramado, Brasil, Pp 150.

Feldman E C, R W Nelson. 1996. Ovarian cycle and vaginal cytology. En: Feldman y Nelson, Canine and Feline Endocrinology and Reproduction. Saunders, Philadelphia, USA, Pp 529-546.

Fidel P L Jr, J R Cutright, C Steele. 2000. Effects of reproductive hormones on experimental vaginal candidiasis. Infect and Immun 68, 651-657

Fidel P L Jr, M Barousse, T Espinosa, M Ficarra, J Sturtevant, D H Martin, A J Quayle, K Dunlap. 2004. An Intravaginal Live Candida Challenge in Humans Leads to New Hypotheses for the Immunopathogenesis of Vulvovaginal Candidiasis. Infect and Immun, 2939-2946.

Greene C E, F W Chandler. 1998. Candidiasis. En: Infectious diseases of dog and cat. Saunders, Philadelphia, USA, Pp 414-417.

Guillot J, R Chermette, E Gueho. 1994. Prevalence du genre Malassezia chez les mammiferes. J Mycol Méd 4, 72-79.

Guillot J, E Guého, M Lesourd, G Midgley, G Chévrier, B Dupont. 1996. Identification of Malassezia species: a practical approach. J Mycol Med 6, 103-110.

Gyaurgieva O H, T S Bogomolova, G I Gorshkova. 1996. Meningitis caused by Rhodotorula rubra in an HIV-infected patient. $J$ Med Vet Mycol 34, 357-359.

Hafez E S E. 1995. Reprodução Animal. Manole, São Paulo, Brasil, Pp 582

Hazen K C. 1995. New and emerging yeast pathogens. Clin Microb Review 8, 462-478.

Jeffcoate I. 1998. Physiology and Endocrinology of the bitch. En: BSVA Manual of small animal reproduction and neonatology. British Small Animal Veterinary Association, U K, Pp 1-9.

Jöchle W, A C Andersen. 1977. The estrous cycle in the dog: a review Theriog 7, 113-140.

Lacaz C S, E Porto, J E C Martins, E M Heins-Vaccari, N T Melo. 2002. Tratado de Micologia Médica. Sarvier (eds), São Paulo, Brasil, Pp 1104.

Linares L M, C Marín. 1978. Frequency of yeasts of the genus Candida in humans as pathogens and as part of normal flora. Proceeding of the IV International Conference on the Mycoses. Pan American Health Organization, Washington, USA, Pp 124.

Linde C, I Karlsson. 1984. The correlation between the cytology of the vaginal smear and the time of ovulation in the bitch. $J$ Small An Pract 25, 77-82.
Lui A Y, G S Turett, D L Karter, P C Bellman. 1998. Amphotericin B lipid complex therapy in an AIDS patient with Rhodotorula rubra fungemia. Clin Infect Dis, 892-893.

Mialot J P. 1988. Patologia da reprodução dos carnívoros domésticos. A Hora Veterinária, Pp 160.

Moretti A, B Posteraro, L Boncio, L Mechelli, E D Gasperis, F Agnetti, M Raspa. 2004. Diffuse cutaneous candidiasis in a dog. Diagnosis by PCR-REA. Rev Iberoam Micol 21, 139-142.

Moretti A, L Boncio, B Posteraro, L Mechelli, M Balducci, G Fadda, M La Sorda, M Di Chio, V Grelloni, F Agnetti. 2006. Co-cutaneous infection in a dog. PCR-reverse identification of Candida tropicalis on skin biopsy. J Mycol Médicale 16, 30-36.

Nascente P S. 2001. Malassezia pachydermatis em cães e gatos: estudo da freqüência e avaliação da sensibilidade aos antifúngicos cetoconazol, fluconazol e itraconazol. Dissertação Mestrado. Faculdade de Veterinária. Universidade Federal de Pelotas.

Oliveira C M, E O Costa, J A P Silva.1998. Microbiota aeróbica em fêmeas caninas hígidas durante o ciclo estral. Avaliação da sensibilidade aos antimicrobianos. Rev Bras Med Vet 20, 78-84.

Olson P N, T M Nett. 1986. Reproductive endocrinology and physiology of the bitch. En: Morrow D A. Current therapy in theriogenology. Saunders, Philadelphia, USA, Pp 453-457.

Papadogeorgakis H, E Frangoulis, A Katsambas. 1999. Rhodotorula rubra fungaemia in an immunosuppressed patient. J Eur Acad Dermat Vener 12, 169-179.

Plant J D, W S Rosenkrantz, C E Griffin. 1992. Factors associated with and prevalence of high Malassezia pachydermatis number on dog skin. J Amer Vet Med Assoc 201, 879-882.

Raposo J B, M O Nobre, C G Fernandes, M Porto. 1996. Candidíase cutânea em um canino. Rev Fac Zootec, Vet e Agron 2/3, 11-14.

Ribeiro M A, R Dietze, C R Paula, D A Matta, A L Colombo. 2001. Susceptibility profile of vaginal yeast isolates from Brazil. Mycopath 151, 5-10.

Rippon J N. 1988. The pathogenic fungi and the pathogenic actinomycetes. En: Medical Mycology. Saunders, Philadelphia, USA, Pp 797

Rodrigues B A, J L Rodrigues. 2002. Endocrinologia reprodutiva na cadela. Rev Clin Vet 40, 50-58.

Rodriguez M C, C Sotomayor, M E Costamagna, A M Cabanillas, B S Renteria, A M Masini, S Correa. 2003. Immunocompetence of macrophages in rats exposed to Candida albicans infection and stress. Amer J Physiol Cell 284, 111-118.

Roszel J F. 1975. Genital cytology of the bitch. Vet. Scope 19, 12-15.

Rusthoven J J, R Feld, P G Tuffnell. 1984. Systemic infection by Rhodotorula spp in the immunocompromised host. $J$ Infect 8, 241-246.

Sidrim J J C, J L B Moreira. 1999. Fundamentos clínicos e laboratoriai da micologia médica. Guanabara Koogan, Rio de Janeiro, Pp 287.

Sierra P, J Guillot, H Jacob, S Bussieras, R Chermette. 2000. Fungal flora on cutaneous and mucosal surfaces of cats infected with feline immunodeficiency virus or feline leukemia virus. Amer J Vet Res $61,158-161$

Silva J O, S A Franceschini, R C Candido. 2002. Presença de leveduras em mucosas e fezes de indivíduos aparentemente saudáveis e de pessoas com sintomas de infecção fúngica. Rev Inst Adolfo Lutz 61, 113-120.

Watts J R, P J Wright, K C Whithear. 1996. Uterine, cervical and vaginal microflora of the normal bitch throughout the reproductive cycle. J Small An Pract 37, 54-60.

Wira C R, R M Rossoll, C Kaushic. 2000. Antigen-presenting cells in the female reproductive tract: Influence of estradiol on antigen presentation by vaginal cells. Endocr 141, 2877-2885.

Valtonen M, L Jalkanen. 1993. Species-specific features of oestrou developement and blastogenesis in domestic canine species. $J$ Reprod Fert supplemment 47, 133-137.

Vannucchi C I, S Satzinger, S E C Santos. 1997. Técnica de citologia vaginal como método de diagnóstico da fase do ciclo estral em cadelas. Rev Clin Vet 9, 14-19.

Verstegen J. 1999. Hormonal cycle and vaginal cytology in the bitch. In: Reproduktion hos hund. Dansk veterinaerforening for Husdyrreproduktion. Proceedings. Escandinávia, Pp 7-21.

Ziarrusta G B. 2002. Vulvovaginitis candidíasica. Rev Iberoam Micol 19, 22-24. 